\title{
Perspective
}

PERSPECTIVE Actualité en histoire de l'art

2| 2009

L'Espagne

\section{Historiographie et esprit de clocher : l'art espagnol de l'époque moderne et ses rapports à l'art étranger}

Historiography and provincialism: relations between Spanish and foreign art in the Early Modern period

\section{Fernando Checa}

\section{OpenEdition}

Journals

Édition électronique

URL : http://journals.openedition.org/perspective/1363

DOI : $10.4000 /$ perspective. 1363

ISSN : 2269-7721

\section{Éditeur}

Institut national d'histoire de l'art

Édition imprimée

Date de publication : 30 juin 2009

Pagination : 207-214

ISSN : 1777-7852

Référence électronique

Fernando Checa, « Historiographie et esprit de clocher : l'art espagnol de l'époque moderne et ses rapports à l'art étranger », Perspective [En ligne], 2 | 2009, mis en ligne le 22 juillet 2014, consulté le 01 octobre 2020. URL : http://journals.openedition.org/perspective/1363 ; DOI : https://doi.org/10.4000/ perspective.1363 


\section{Historiographie et esprit de clocher : l'art espagnol de l'époque moderne et ses rapports à l'art étranger}

Fernando Checa

L'art espagnol s'étant trouvé confronté dès le début de l'époque moderne aux productions artistiques d'autres centres de création, notamment flamands et italiens, ses historiens ont dû concilier, au sein d'un discours cohérent, une interprétation nationaliste de cet art avec le constat de la présence en Espagne d'un grand nombre $d^{\prime} œ u v r e s$ et d'artistes originaires d'autres pays ou aires culturelles. Les formes successives que revêtit ce débat furent variées et elles infléchirent la réponse donnée à la difficile question de l'existence ou non d'un " art espagnol ». Dès la fin de la Renaissance, le père José de Sigüenza (1544-1602) avait offert une première réponse dans sa chronique où sont narrées les vicissitudes de la construction et de la réalisation du décor de l'Escurial. Dans son Historia de la Orden de San Jerónimo ${ }^{1}$ se trouve affirmée, pour la première fois de manière pleinement consciente, l'existence $d^{\prime}$ 'une manière picturale proprement espagnole : faisant allusion aux tableaux de Juan Fernández de Navarrete el Mudo qui ornaient l'Escurial, Sigüenza compare le style du peintre espagnol à celui des Flamands et des Vénitiens afin de mieux I'en distinguer.

C'est surtout au sein des milieux de la cour qu'apparut et se développa la tradition des échanges artistiques; ainsi commença-t-on à importer les œuvres d'artistes internationaux. Le phénomène, qui s'était déjà produit au cours du Moyen Âge, s'amplifia dès la fin de l'époque médiévale à la cour des Rois Catholiques - où travaillèrent, pour ne citer que les exemples les plus célèbres, des peintres comme Juan de Flandes et Michel Sittow, ainsi que des sculpteurs tels que Torrigiano ou Domenico Fancelli - et se poursuivit sous Charles Quint et Philippe II, dont les palais s'ornèrent de nombreuses œuvres de Titien, de Jérôme Bosch, de Leone Leoni ou de Pompeo Leoni, sans aller jusqu'à détailler les impressionnantes collections de peintures de Philippe IV, riches en œuvres de Titien, de Tintoret, de Véronèse, de Rubens et plus largement de l'école flamande. Les Bourbons, nouvelle dynastie sur le trône d'Espagne, maintinrent cette tradition : il suffit de rappeler qu'au milieu du XVIII' siècle, les deux peintres les plus célèbres d'Europe, Giovanni Battista Tiepolo et Antón Rafael Mengs, rivalisaient de talent sur les fresques du nouveau Palacio Real madrilène, dont I'Italien Filippo Juvarra avait élaboré le projet architectural. Cet ensemble de fresques, qui n'a pas encore été étudié en tant que tel, compte parmi les plus spectaculaires de l'époque.

Hors de la cour, l'engouement pour les œuvres d'artistes étrangers fut également sensible, quoique de moindre ampleur. L'un des artistes qui définirent I' " école picturale espagnole», Domenicos Theotocopoulos ou El Greco, était né en Crète et avait reçu sa formation de peintre dans l'Italie de la fin de la Renaissance, à Venise et à Rome. Gardons-nous d'oublier, de même, que la présence d'artistes flamands fut continuelle dans I'Espagne du XVI $l^{\mathrm{e}}$ siècle. 

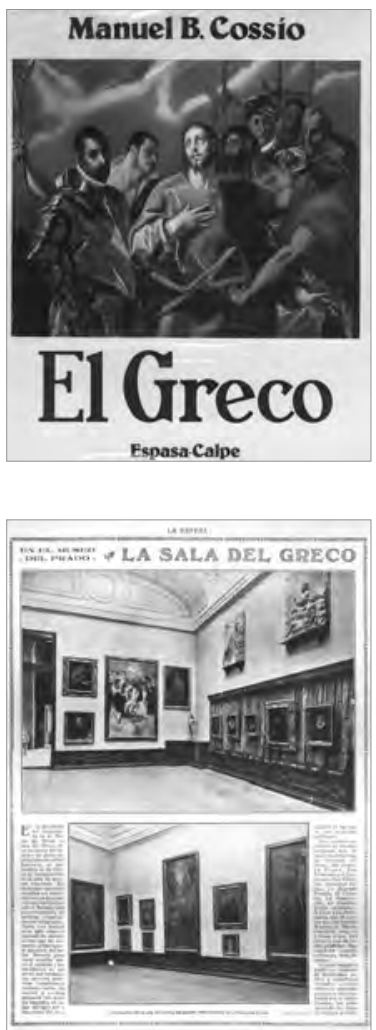

1. Manuel B. Cossío, El Greco, Madrid, (1908) 1984

2. Article sur la salle du Greco au Museo Nacional del Prado, dans La Esfera, 351, 25 septembre 1920 [Leticia Ruiz Gómez, El Greco, Madrid, 2007, p. 23].
Ce phénomène, dont nous avons seulement esquissé les contours, est l'un des plus caractéristiques de I'histoire de l'art espagnol du XVI au XVIII ${ }^{\mathrm{e}}$ siècle. II fut aussi l'un des problèmes les plus aigus auxquels furent confrontés les historiens de "l'art espagnol », dont la discipline fut soumise, dès le XIXe siècle mais surtout durant les quatre-vingts premières années du $\mathrm{XX}^{\mathrm{e}}$ siècle, à des préoccupations résolument nationalistes.

L'exemple du Greco est hautement significatif. Son œuvre sombra dans un oubli presque complet après sa mort à Tolède en 1614 et il faut attendre la fin du XIX siècle pour qu'il connaisse un regain d'intérêt d'une grande force, sous l'effet de deux séries de facteurs : d'une part, l'influence des mouvements artistiques d'avant-garde comme le modernisme ou l'expressionnisme, et l'admiration dont témoignèrent des artistes tels que Picasso pour ses peintures ; d'autre part le surgissement d'un nationalisme espagnol - ou plutôt castillan - de type introspectif ( $I^{\prime}$ « intrahistoire » affirmée de Miguel de Unamuno dans son essai fondamental En torno al casticismo de $1902^{2}$ ), caractéristique de la génération dite « de 98 ».L'un des plus remarquables ouvrages historiographiques espagnols du XXe siècle, El Greco de Manuel B. Cossío, paru en 1908, cristallisa cette approche : le Crétois, dont la figure est interprétée à la lumière de la littérature mystique espagnole du XVI siècle, y est considéré comme l'un des meilleurs représentants, sinon le meilleur, d'une âme espagnole " éternelle» (fig. 1$)^{3}$.

Cette interprétation se maintint presque jusqu'à nos jours, avec plus ou moins de nuances, et $c^{\prime}$ est depuis quelques années seulement que la découverte et I'analyse des idées esthétiques du peintre ${ }^{4}$, I'attention portée à son étape italienne ${ }^{5}$, une approche de son œuvre intégrée à de vastes perspectives historiographiques ${ }^{6}$, ou encore l'étude critique de sa réception par les cercles artistiques d'avant-garde au début du $\mathrm{XX}^{\mathrm{e}}$ siècle ${ }^{7}$, situent sa production dans une perspective plus adéquate, comme c'est le cas du récent catalogue consacré à ses œuvres conservées au Museo del Prado (fig. 2) ${ }^{8}$.

$\mathrm{L}^{\prime}$ « appropriation nationaliste» d'une figure telle que le Greco constitue sans nul doute un cas historiographique spectaculaire, dans la mesure où elle s'est produite à une époque particulièrement sensible à ces questions. Au début du $X X^{e}$ siècle, un grand nombre d'intellectuels espagnols, madrilènes pour la plupart, s'était jeté dans la quête de caractéristiques atemporelles de "lo hispano »le proprement hispanique. Toutefois, le traitement historiographique de la peinture du Greco n'est pas unique. À la suite de la parution de la monographie déjà ancienne de Diego Angulo en 1951, il a fallu attendre 2008 pour qu'une analyse actualisée de I'œuvre de Pedro de Campaña - non exempte, hélas, de manifestations d'un esprit de clocher sévillan - soit proposée par Enrique de Valdivieso ${ }^{9}$. Nicole Dacos, dans de nombreux articles consacrés à ce peintre, a également offert une nouvelle approche de sa production, insérée dans une perspective plus internationale ${ }^{10}$.

La présence de riches collections de tapisseries et de peintures flamandes en Espagne du XVe au XVII siècle constitue un autre exemple significatif de la difficulté des historiens à accepter la part d'étranger dans l'art espagnol. En effet, jusqu'à très récemment, aucun effort réel n'a été fait pour intégrer ces ensembles à un discours cohérent sur histoire des collections en Espagne ${ }^{11}$. Certains historiens de l'art jugent même surprenant que des œuvres majeures comme les Époux Arnolfini de Van Eyck, I'Adoration des bergers de Van der Goes ou le polyptyque dit " de Miraflores » de Van der Weyden aient fait partie des collections espagnoles jusqu'à des dates très avancées (I'invasion napoléonienne ou le XIXe siècle). Les collections du Museo del 
Prado, on le sait, comptent, aujourd'hui encore, de nombreuses œuvres flamandes. Toutefois, dans le cadre d'une historiographie marquée par une posture clairement nationaliste - comme ce fut longtemps le cas de l'historiographie espagnole -, il fut toujours difficile d'intégrer ces phénomènes à un discours attentif à leur contexte, si bien qu'ils ne purent être étudiés que dans des catalogues ${ }^{12}$ ou dans des monographies spécialisées couvrant des thématiques plus larges ${ }^{13}$.

Il en va de même pour le XVII siècle. Nul n'ignore l'extraordinaire importance des tableaux de Rubens et de son école au sein des collections du Prado et, pourtant, après la publication des catalogues de Matías Diaz Padrón en 1975, il a fallu attendre 1999 pour pouvoir lire la première étude globale et contextuelle de cet ensemble de peintures (fig. 3) ${ }^{14}$ ou pour voir ces œuvres analysées à la lumière de leur contexte, la culture visuelle de l'art baroque de cour, lors de l'exposition Velázquez, Rubens, Van Dyck (1999) organisée par Jonathan Brown au Prado ${ }^{15}$.

L'influence de la peinture italienne en Espagne et son incidence sur les débats artistiques espagnols ont fait l'objet d'un traitement historiographique très similaire. Si ce phénomène revêt une grande importance dès le Moyen Âge - chez des artistes comme Dello Delli ou Gherardo Starnina -, c'est à partir du XVI $\mathrm{I}^{\mathrm{e}}$ siècle qu'il devient incontournable et détermine l'évolution de l'art espagnol : les collections espagnoles comptent alors de nombreux tableaux de Titien et d'autres artistes de l'école vénitienne, tandis qu'au $X V I I^{e}$ siècle, celles de Philippe IV s'enrichissent des productions de Raphaël et d'un vaste ensemble de peintures italiennes baroques. Or la réaction historiographique à ce phénomène a été discrète et tardive. L'un des rares ouvrages qui lui aient été consacrés est celui de Pedro Beroqui, Tiziano en el Museo del Prado, publié en $1925^{16}$. L'influence de la peinture vénitienne sur l'art espagnol de la Renaissance ne fait l'objet d'études systématiques que depuis les années 1990 : citons ici les travaux de Fernando Checa et de Matteo Mancini, ainsi que les expositions dont Miguel Falomir fut le commissaire au Museo del Prado, Los Bassano (2001) et Tiziano (2003) ${ }^{17}$.

La présence continuelle d'artistes étrangers en Espagne - à la cour principalement - et ses conséquences historiques ont été soulignées par Miguel Morán Turina à I'occasion de I'exposition qu'il organisa à Madrid en 2002-2003, El arte en la corte de Felipe V (fig 4) ${ }^{18}$. II signala alors que des historiens comme Juan Antonio Gaya Nuño, Enrique Lafuente Ferrari ou Alfonso Pérez Sánchez ne font pas mention dans leurs travaux d'artistes tels que MichelAnge Houasse, Jean Ranc, Michel van Loo ou Andrea Procaccini ; Pérez Sánchez, il est vrai, cite I'œuvre de peintres italiens comme Bartolomé Carducho ou Angelo Nardi qui travaillaient à la cour au début du $\mathrm{XVII}{ }^{\mathrm{e}}$ siècle, mais il ne fait aucune référence aux œuvres de leurs successeurs étrangers à la cour bourbonnaise. Or, ces derniers, selon Morán, présentaient un profil similaire : «des artistes étrangers, qui ont apporté un type de peinture différent de celui qui se pratiquait alors, qui ont travaillé longtemps à la cour et dont la production, prise en exemple, eut d'importantes conséquences sur le futur de l'art espagnol ${ }^{19}$.

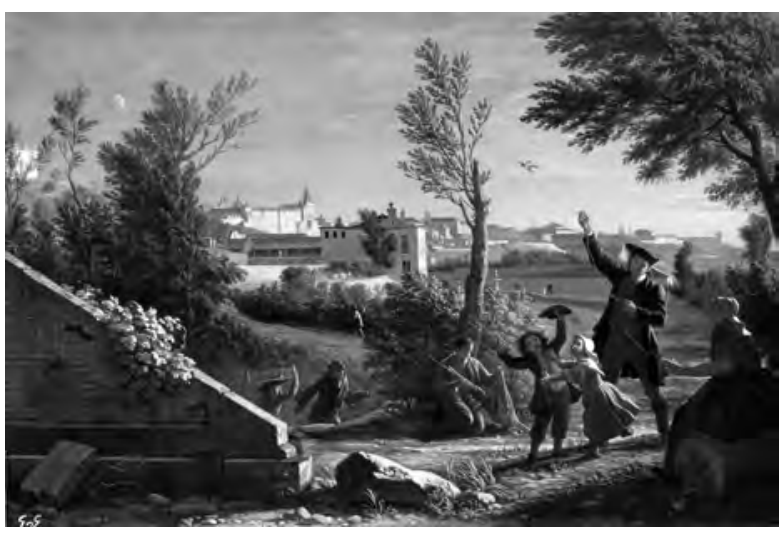

4. Michel-Ange Houasse, Vue de l'Alcazar et une partie de Madrid depuis la montagne du Príncipo Pío, 1715-1727, Ségovie, Palacio Real de La Granja de San Ildefonso [El arte en la corte de Felipe V, Madrid, 2002, p. 210]. 
L'interprétation nationaliste de l'art produit en ou pour l'Espagne à l'époque moderne s'est maintenue durant une période exceptionnellement longue puisqu'elle a perduré jusqu'à la mort de Franco en 1975, au moment où l'historiographie internationale, sur laquelle soufflait déjà un vent nouveau, s'ouvrait à des explications neuves et variées. L'isolement de l'Espagne ne fut donc pas seulement politique, mais aussi culturel et historiographique, comme l'a affirmé Enrique Lafuente Ferrari, alors que la période franquiste touchait à sa fin. Son prologue à la traduction espagnole des Essais d'iconologie d'Erwin Panofsky, premier livre de cet auteur traduit en espagnol ${ }^{20}$, révulsa les représentants d'une historiographie péninsulaire alors moribonde.

Nul ouvrage n'est plus caractéristique de l'historiographie espagnole de l'époque franquiste que la monumentale série intitulée Ars Hispaniae, dont les nombreux tomes, parus au cours des années 1950 et 1960, furent rédigés par les grands historiens du moment (Diego Angulo, Fernando Chueca, José María de Azcárate, Lepoldo Torres Balbás, George Kubler, Francisco Javier Sánchez Cantón...). Dans leur ensemble, ces volumes prolongent jusqu'à une date très avancée les méthodes du positivisme historiographique de la première moitié du XX⿳亠丷厂 siècle et proposent une vision clairement nationaliste de l'art espagnol. Chacun d'eux aborde son thème respectif à travers une division par tiers de siècle et distingue, selon la méthode géographique des positivistes du XIXe siècle, des écoles régionales. En marge de cette collection mais composé par I'un des plus illustres historiens qui y collaborèrent, les Invariantes castizos de la arquitectura española de Fernando Chueca ${ }^{21}$ offrent une excellente synthèse de ce que fut l'interprétation nationaliste de l'art espagnol au milieu du XXe siècle. L'esprit d'une œuvre comme I'Historia del arte español de Fernando Jiménez Placer ${ }^{22}$ a été résumé comme suit par Miguel Morán, qui se réfère ici à l'art produit à et pour la cour sous Philippe $V:$ : Tout le discours de Jiménez-Placer [...] tourne autour de deux axes, qui sont de parfaits lieux communs : il évoque d'une part les effets pernicieux des 'influences exotiques' introduites par la pléiade d'artistes étrangers que les rois 'importent à grande échelle' et qui finissent par anéantir I"originalité personnelle' de 'notre grande peinture nationale' ; il affirme d'autre part la traditionnelle imperméabilité de l'art espagnol à tout ce qui est étranger à sa profonde originalité, au caractère individualiste de ses peintres et à la véritable essence du génie espagnol ${ }^{23}$. En 1969 encore, un critique comme José María Moreno Galván, nullement suspect sur le plan politique, justifiait en ces termes le peu d'attention que I'historien Manuel Gómez-Moreno avait porté à la statuaire classique : "Cet intérêt peu soutenu de Gómez Moreno pour la statuaire classique est bien compréhensible : elle était étrangère à son être profond et je déclare, me présentant une fois encore comme son disciple, qu'elle est étrangère au mien. Être espagnol peut bien être un hasard. Mais l'être sincèrement exige de n'être pas un classique ${ }^{24}$.

$\mathrm{C}^{\prime}$ est dans cette perspective, et en tenant compte du prolongement, jusque dans la seconde moitié du XXe siècle, du discours historiographique nationaliste autour de I'" essence» de l'art espagnol, qu'il faut comprendre les hésitations et contradictions d'historiens de l'époque. Ces derniers eurent la tâche ardue d'intégrer les principaux événements artistiques que connut I'Espagne du XV au $X V I I I^{e}$ siècle à un discours plus général, qui tienne compte du contexte européen et de son influence considérable sur le développement même de l'art produit en ou pour l'Espagne durant cette période.

Tout ceci trouve son explication dans deux causes principales. La première, que nous avons déjà signalée, a trait au nationalisme politique et historique espagnol au sein duquel nous pourrions distinguer deux grands moments : les premières années 
du $X X^{e}$ siècle d'une part - avec une prolongation dans les travaux d'auteurs comme Lafuente Ferrari et, de manière plus surprenante, dans des expositions récemment organisées au Museo del Prado, ou encore celle au Musée Guggenheim de New York, soutenue par le gouvernement espagnol ${ }^{25}$-, et la période franquiste d'autre part. La seconde cause, liée à la première, concerne le développement en Espagne de I'histoire de l'art comme discipline, dont les principaux repères historiographiques proviennent d'études biographiques ou de dictionnaires d'artistes espagnols.

À l'orée du XVII ${ }^{e}$ siècle, comme on l'a vu, le père Sigüenza ne faisait aucune difficulté pour débattre de ce qu'il considérait comme les traits caractéristiques de la peinture espagnole de cour, ni pour inclure dans son discours, fort proche de celui de Vasari, des peintres comme Titien, Léonard de Vinci, Jacopo Bassano, Corrège, Jérôme Bosch ou Michel de Coxcie. De même, à une époque - le XVII siècle où les artistes espagnols, y compris Diego Velázquez, jugeaient exemplaires les peintures du Titien ou de Rubens qu'ils pouvaient voir en nombre à la cour, des auteurs de traités tels que Vicento Carducho ou Francisco Pacheco discutaient des mérites des artistes étrangers et mentionnaient leurs œuvres de la manière la plus naturelle qu'il fût. Antonio Acisclo Palomino fit de même dans la première série

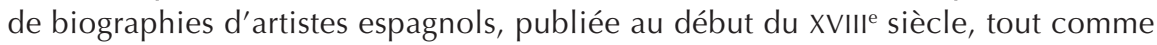
Juan Agustín Ceán Bermúdez, qui n’hésita pas à inclure dans son Diccionario histórico de los más ilustres profesores de las Bellas Artes en España ${ }^{26}$, parmi celles de beaucoup d'autres artistes étrangers, les biographies de Titien, de Rubens et de Giovanni Battista Tiepolo - tous peintres qui ne tardèrent pas à se voir exclus des ouvrages généraux d'histoire de l'art espagnols -, en soulignant l'excellence de leurs œuvres conservées en Espagne. II en va de même du Viaje de España d'Antonio Ponz, autre pilier de la construction du discours sur l'histoire de l'art espagnol (fig. 5) ${ }^{27}$.

La situation commença à changer au cours du XIXe siècle, au moment où le discours romantique de type nationaliste se trouva renforcé par une tendance à I'isolationnisme politique et culturel qui atteint son paroxysme à la fin du XIX et au début du XXe siècle. Restée pour l'essentiel en marge de la société industrielle du $X I X$ e siècle, I'Espagne se transforma en "le pays romantique par excellence », dont on valorisa de préférence ce que la culture de l'époque, en Espagne et ailleurs, considérait comme " autochtone » et " exotique ». Ainsi, en cette fin de XIXe siècle, certains auteurs majeurs tels que Marcelino Menéndez y Pelayo revendiquèrent, dans une perspective réactionnaire, tout ce qu'ils estimaient être proprement espagnol, c'est-à-dire vierge de toute contamination étrangère. Seul un petit nombre d'institutions parvint à maintenir un certain équilibre, parmi lesquelles le Museo del Prado. Fondé en 1819 à partir de la très internationale collection royale espagnole de peinture, il s'imposa néanmoins dès le début comme un monument à l'école espagnole, avec Velázquez, Murillo et, plus tard, Goya en tête. Toutefois, la galerie centrale, l'espace le plus important du musée, abrita la peinture italienne pendant presque tout le XIXe siècle et ne s'ouvrit à l'école espagnole qu'au début du XXe siècle, bien que les contacts avec l'actualité muséographique européenne, et en particulier parisienne, aient été maintenus tout au long du XIX siècle grâce aux membres de la famille Madrazo.

La crise espagnole de la fin du XIXe siècle et la dictature prolongée qui s'instaura après la guerre civile expliquent en grande partie l'état de l'historiographie en Espagne. Il a fallu attendre le changement politique et culturel du dernier quart du $X x^{e}$ siècle pour voir émerger une nouvelle approche de la discipline,

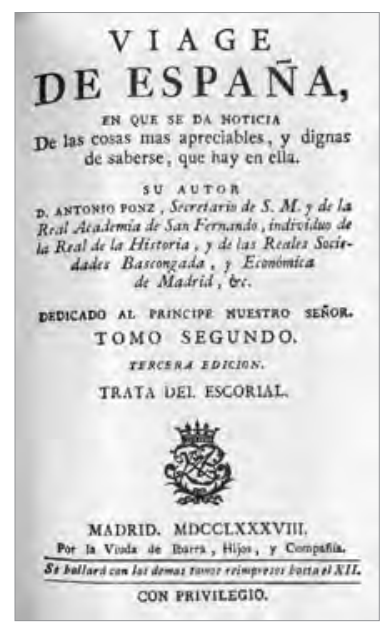

5. Frontispice d'Antonio Ponz, Viage de España, II, Trata del Escorial, Madrid, 3e éd., 1788. 

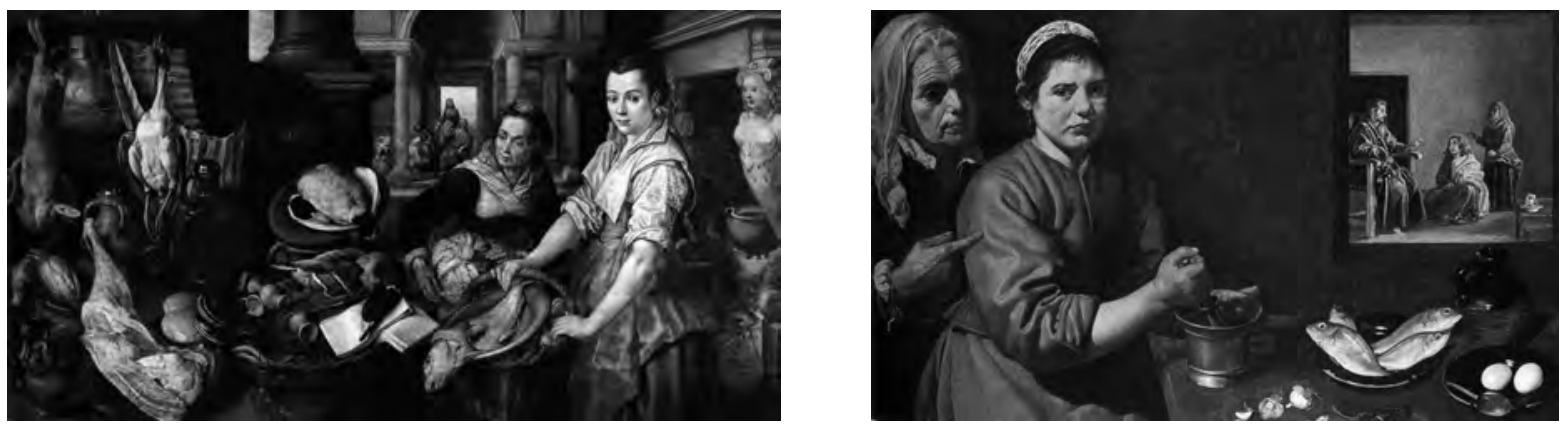

6. Le Christ chez Marthe et Marie : a. Joachim Beuckelaer, 1568, Madrid, Museo Nacional del Prado ; b. Diego Velázquez, 1618, Londres, The National Gallery [Velázquez's Fables. Mythology and Sacred History in the Golden Age, Madrid, 2007, p. 114-117]. enfin à même de replacer l'art espagnol dans son contexte européen, et ainsi voir réintégrer à son discours cet aspect fondamental de l'histoire de l'art péninsulaire.

Nous ne pouvons néanmoins nous féliciter de toutes les conséquences de cette évolution. S'il est vrai qu'on ne lit plus guère $d$ 'histoires monolithiques de l'art espagnol, on a en revanche vu surgir, à la faveur du développement des diverses autonomies régionales, une kyrielle d'études et d'ouvrages locaux d'histoire de l'art, d'où l'essor d'une historiographie locale le plus souvent à courtes vues et rétive à reconnaître toute influence "extranjerizantes ». Au nationalisme espagnol a donc succédé le nationalisme des communautés autonomes.

Quoi qu'il en soit, la nouvelle génération d'historiens a découvert dans I'histoire de l'art un champ offrant la possibilité d'une rénovation méthodologique et d'une ouverture à de vastes horizons internationaux. Ainsi, une compréhension renouvelée de la peinture italienne, flamande et française - en plus de la seule peinture espagnole - a été rendue possible par des expositions comme El palacio del Rey Planeta: Felipe IV y el Buen Retiro en $2005^{28}$, dont le commissaire Andrés Úbeda de los Cobos est également l'auteur d'une excellente monographie consacrée au séjour espagnol d'un autre grand oublié, Luca Giordano, publiée à I'occasion d'une exposition au Museo del Prado en $2008^{29}$.

Les conséquences de ces changements ont également été bénéfiques à l'étude et à la compréhension de l'œuvre d'artistes nés en Espagne, comme Velázquez luimême. De ce point de vue, les travaux de Jonathan Brown, en soulignant le rôle que jouèrent les collections royales espagnoles dans la vie de l'artiste comme " peintre et homme de cour », furent d'une importance décisive ${ }^{30}$. Sans eux, des expositions comme celle sur le Buen Retiro déjà citée ou Fábulas de Velázquez, également organisée au Prado par Javier Portús en 2007-2008²1, où I'œuvre du Sévillan se trouvait confrontée à celle d'autres artistes espagnols et étrangers (fig. 6), n'auraient sans doute jamais vu le jour.

Il semble donc qu'en dépit des difficultés signalées, l'histoire de l'art espagnole marche désormais sur la bonne voie, une voie internationale. 
1. José de Sigüenza, Historia de la Orden de San Jerónimo, Salamanque, (1605) 2005.

2. Miguel de Unamuno, En torno al casticismo, Madrid, (1895) 1902 [trad. fr. : L'Essence de l'Espagne, Paris, (1923) 1999.

3. Manuel B. Cossío, El Greco, Madrid, 1908.

4. Xavier de Salas, Fernando Marías, El Greco y el arte de su tiempo. Las notas de El Greco a Vasari, Tolède, 1992.

5. Jonathan Brown, "El Greco y Toledo", dans El Greco de Toledo, (cat. expo., Madrid, Museo Nacional del Prado, 1982), Madrid, 1982, p. 75147 ; Nicos Hadjanicolau éd., El Greco in Italy and Italian Art, (colloque, Crète, 1995), Réthymnon, 1999.

6. José Alvarez Lopera, De Ceán a Cossío; la fortuna crítica del Greco en el siglo XIX, Madrid, 1987.

7. El Greco. Su revalorización por el modernismo catalán, (cat. expo., Barcelone, Museu Nacional d'Art de Catalunya, 1996-1997), Barcelone, 1996 ; El Greco. Toledo 1900, (cat. expo., Séville, Museo de Bellas Artes de Sevilla, 2008), Madrid 2008.

8. Leticia Ruiz, El Greco en el Museo del Prado. Catálogo razonado, Madrid, 2007.

9. Diego Agulo, Pedro de Campaña, Séville, 1951 ; Enrique Valdivieso, Pedro de Campaña, Séville, 2008.

10. Nicole Dacos, "Peter de Kempeneer - Pedro de Campaña as a draughtsman ", dans Master Drawings, 25/4, 1988, p. 359-389; "Pedro de Campaña dopo Siviglia: Arazzi e altri inediti », dans Bolletino d'arte, 6/8, 1980, p. 1-44.

11. Guy Delmarcel, «Le roi Philippe d’Espagne et la tapisserie. L'inventaire de Madrid de 1598 ", dans Gazette des Beaux Arts, 6/134, 1999, p. 153178 ; Concepción Herrero, Tapices de Isabel la Católica. Orígenes de la colección real española, Madrid, 2004 ; Fernando Checa, Tapisseries flamandes pour les ducs de Bourgogne, l'empereur Charles Quint et le roi Philippe II, Bruxelles, 2009.

12. Elisa Bermejo, La pintura de los primitivos flamencos en España, Madrid, 1980.

13. C'est le cas, par exemple, de l'ouvrage de Roger van Schoute, La chapelle Royale de Grenade, Bruxelles, 1963.

14. Matías Díaz Padrón, Museo Del Prado. Catálogo De Pinturas. I Escuela Flamenca Siglo XVII, 2 vol., Madrid, 1975 ; Alejandro Vergara, Rubens and his Spanish Patrons, Cambridge/New York, 1999.

15. Velázquez, Rubens y Van Dyck: pintores cortesanos del siglo XVII, Jonathan Brown éd., (cat. expo., Madrid, Museo Nacional del Prado, 1999), Madrid, 1999.
16. Pedro Beroqui, Tiziano en el museo del Prado, Madrid, 1925

17. Fernando Checa, Tiziano y la Monarquía hispánica. Usos y funciones de la pintura veneciana en España. Siglos XVI y XVII, Madrid, 1994 ; Matteo Mancini, Tiziano e le corti d'asburgo, Venise, 1998 ; Los Bassano en la España del Siglo de Oro, Miguel Falomir éd., (cat. expo., Madrid, Museo Nacional del Prado, 2001), Madrid, 2001 ; Tiziano, (cat. expo., Madrid, Museo Nacional del Prado, 2003), Madrid, 2003.

18. El arte en la corte de Felipe V, Miguel Morán Turina éd., (cat. expo., Madrid, Palacio Real de Madrid, Museo Nacional del Prado, Casa de las Alhajas, 2002-2003), Madrid, 2002.

19. "...unos artistas extranjeros, que traen un tipo de pintura diferente al que aquí se practica, que trabajan en la corte durante un largo período de tiempo y cuya actividad y ejemplo va a tener consecuencias importantes para el futuro del arte español » (Morán, 2002, cité n. 18, p. 23).

20. Enrique Lafuente Ferrari, "Introducción a Panofsky (Iconología e Historia del arte) », dans Erwin Panofsky, Estudios de iconología, Madrid, 1972, p. IX-XL.

21. Fernando Chueca, Invariantes castizos de la arquitectura española, Madrid, 1947.

22. Fernando Jiménez Placer, Historia del arte español, Barcelone, 1955.

23. "Todo el discurso de Jiménez-Placer [...] gira alrededor de un doble eje, que es absolutamente tópico: por una parte el del efecto pernicioso de esos 'influjos exóticos' traídos por la pléyade de artistas extranjeros que los reyes 'importan en gran escala' y que acaban por matar la 'originalidad personal' de 'nuestra gran pintura nacional' [...] y por otra, la tradicional impermeabilidad del arte español a todo lo que resulta ajeno a su profunda originalidad, al carácter individualista de sus pintores y a las verdaderas esencias de lo español » (Morán, 2002, cité n. 18, p. 24).

24. "Y bien comprensible es este no proseguido interés de Gómez Moreno para con la estatuaria clásica. No era lo suyo, como declarándome una ves más su discípulo, no es lo mío. Ser español puede ser una casualidad. Pero serlo de un modo cordial exige no ser un clásico " (Manuel Moreno Galván, "Ante el centenario de Gómez Moreno. Historia de sus libros ", dans Archivo español de arte, 165, 1969, p. 4).

25. Citons, à titre d'exemple, l'exposition au Museo del Prado consacrée au portrait en Espagne, El retrato español del Greco a Picasso, (cat. expo., Madrid, Museo Nacional del Prado, 2004-2005), Madrid, 2004. Pour celle accueillie par le Guggenheim, voir Pintura española de El Greco a Picasso: el tiempo, la verdad y la historia, 
Carmen Giménez, Francisco Calvo Serraller éd. (cat. expo., New York, Solomon R. Guggenheim Museum, 2006-2007), Madrid, 2006.

26. Antonio Acisclo Palomino, El Parnaso español pintoresco y laureado, Madrid, 1715 ; Juan Agustín Ceán Bermúdez, Diccionario histórico de los más ilustres profesores de las Bellas Artes en España, 6 vol., Madrid, 1800

27. Antonio Ponz, Viaje de España, 18 vol., Madrid, 1772-1794.

28. El palacio del rey Planeta: Felipe IV y el Buen Retiro, Andrés Úbeda de los Cobos éd., (cat. expo. Madrid, Museo Nacional del Prado, 2005), Madrid, 2005. L'ouvrage de référence pour ce thème est Jonathan Brown, John H. Elliott, Un palacio para el Rey: el Retiro y la corte de Felipe IV, Madrid, (1980) 2003.

29. Luca Giordano y el Casón del Buen Retiro, Andrés úbeda de los Cobos éd., (cat. expo., Madrid, Casón del Buen Retiro, 2008), Madrid, 2008.

30. Jonathan Brown, Velázquez, Painter and Courtier, New Haven, 1986.

31. Fábulas de Velázquez. Mitología e historia sagrada en el Siglo de Oro, Javier Portús éd., (cat. expo., Madrid, Museo Nacional del Prado, 20072008), Madrid, 2007 [éd. angl. : Velázquez's Fables. Mythology and Sacred History in the Golden Age, Madrid, 2007]. 